SIT-HEP/TM-18

\title{
Curvaton paradigm can accommodate multiple low inflation scales
}

\author{
Tomohiro Matsuda ${ }^{1}$ \\ Laboratory of Physics, Saitama Institute of Technology, \\ Fusaiji, Okabe-machi, Saitama 369-0293, Japan
}

\begin{abstract}
Recent arguments show that some curvaton field may generate the cosmological curvature perturbation. As the curvaton is independent of the inflaton field, there is a hope that the fine-tunings of inflation models can be cured by the curvaton scenario. More recently, however, D.H.Lyth discussed that there is a strong bound for the Hubble parameter during inflation even if one assumes the curvaton scenario. Although the most serious constraint was evaded, the bound seems rather crucial for many models of a low inflation scale. In this paper we try to remove the constraint. We show that the bound is drastically modified if there were multiple stages of inflation.
\end{abstract}

\footnotetext{
${ }^{1}$ matsuda@sit.ac.jp
} 


\section{Introduction}

It has recently been proposed that the energy density perturbations could originate in a scalar field other than the conventional inflaton field [1, 2], which is called curvaton. After inflation, the curvaton starts to oscillate in a radiation background. During this period the energy density of the curvaton grows to account for the cosmological curvature perturbation when it decays. This curvaton paradigm has attracted a lot of attention because it has an obvious advantage. The inflaton energy scale is decoupled from the magnitude of the cosmic microwave background(CMB) temperature fluctuations, which may cure the fine-tunings of inflation models. Along this line of thought, it seems attractive to suppose that the curvaton paradigm can also save the models of a low inflation scale[3.

The construction of a realistic model of low inflation scale is an interesting problem especially when the fundamental scale is (much) lower than the Planck scale, because in these models the Hubble parameter cannot become so large as the one in the conventional models of inflation. In the history of the string theory, originally the inverse of the size of extra dimensions as well as the fundamental scale was assumed to be as large as $M_{p}$. However, later observations showed that there is no reason to require such a tiny compactification radius [4]. In models of large extra dimensions, the observed Planck mass is obtained by the relation $M_{p}^{2}=M_{*}^{n+2} V_{n}$, where $M_{*}$ and $V_{n}$ denote the fundamental scale of gravity and the volume of the $n$-dimensional compact space. In the new scenario of the string theory, the compactification radius and the fundamental scale are unknown parameters that should be determined by observations. However, in many cases the cosmology of the large extra dimension must be quite different from the conventional ones. $^{2}$

In spite of the above expectations, D.H.Lyth showed recently that there will be a strong bound for the Hubble parameter during inflation even if one assumes curvaton scenario 10. At the same time, a mechanism that evades the most serious constraint is

\footnotetext{
${ }^{2}$ Finding crucial constraints on the compactification, or constructing successful models of the inflation with low (or intermediate) fundamental scale is a challenging issue $[5]$. In the models of large extra dimensions, the difficulties of inflation are sometimes related to the stability of the compactified space [5] or the mechanism of baryogenesis that must take place after inflation [7, 8, 9].
} 
also suggested in ref. 10. However, to achieve the bound $H_{I}>10 \mathrm{TeV}$, a huge curvaton mass $\left(m_{\sigma} \sim M_{p}\right)$ is required after the phase transition that takes place at the end of inflation. Moreover, since the obtained bound is rather restrictive $\left(H_{I}>10 T e V\right)$, it should be fair to say that many models of a low inflation scale are still not liberated by the curvaton 3$]$.

In this paper we show that the constraints obtained in [10] are relaxed to a satisfactory level if there were multiple stages of inflation. No large hierarchy between the scales of each inflation is required. At least two stages of inflation are required in our scenario. Denoting the vacuum energy during the two kinds of inflation by $V_{1}$ and $V_{2}$, the original constraint is reduced by the factor of $\epsilon^{6}$, where $\epsilon$ is the ratio of the scales, $\epsilon \equiv\left(V_{2} / V_{1}\right)^{\frac{1}{4}}$. As a result, for example, if the original bound for single inflation is $H_{I}>10^{7} \mathrm{GeV}$, we can reduce it as $H_{1}>10^{-5} \mathrm{GeV}$ for $\epsilon=10^{-2}$, where $H_{1}$ is the Hubble parameter during the first inflation. No sensible bound is obtained for the Hubble parameter during the second inflation.

\section{Constraint on inflation scales}

Here it may be helpful to begin with the review of the discussion in ref. [10]. First we start with more general settings and try to reproduce the constraint showing how the original model in [10] is realized in the boundary condition of our settings. For simplicity, we assume that the curvaton field $\sigma$ is frozen at $\sigma=\sigma_{\text {osc }}$ from the epoch of horizon exit during first inflation to the epoch when the curvaton start to oscillate. At the time when the curvaton starts to oscillate, its density is $\rho_{\sigma} \sim m_{\sigma}^{2} \sigma_{\text {osc }}^{2}$. Denoting the total density of other fields by $\rho_{\text {tot }}$, the ratio $r$ at the time of curvaton oscillation is

$$
\left.\frac{\rho_{\sigma}}{\rho_{t o t}}\right|_{H=H_{o s c}} \sim \frac{m_{\sigma}^{2} \sigma_{o s c}^{2}}{H_{o s c}^{2} M_{p}^{2}} .
$$

During the period when the total density $\rho_{t o t}$ is radiation-dominated, the ratio $r$ grows and reaches finally at

$$
r \leq \frac{\sqrt{H_{o s c} M_{p}}}{T_{d}} \frac{m_{\sigma}^{2} \sigma_{o s c}^{2}}{H_{o s c}^{2} M_{p}^{2}}
$$


when the curvaton decay. Here $T_{d}$ is the temperature just after curvaton decay. Now the curvature perturbation

$$
\zeta \simeq \frac{r}{3} \frac{\delta \rho_{\sigma}}{\rho_{\sigma}}
$$

is generated by the curvaton. Using the spectrum of the perturbation $<\delta \sigma_{\text {osc }}^{2}>=\left(\frac{H_{I}}{2 \pi}\right)^{2}$, the spectrum of the curvature perturbation is given by

$$
\mathcal{P}_{\zeta}^{\frac{1}{2}} \simeq \frac{2 r}{3} \frac{H_{I}}{2 \pi \sigma_{o s c}}
$$

Using the required value from the observations $\mathcal{P}_{\zeta}^{\frac{1}{2}}=5 \times 10^{-5}$, one obtains that

$$
\frac{2 r}{3} \frac{H_{I}}{2 \pi \sigma_{\text {osc }}} \simeq 5 \times 10^{-5}
$$

Using (2.2) and (2.5), one finds the following constraint;

$$
\frac{2}{3} \frac{H_{I}}{2 \pi \sigma_{o s c}} \frac{\sqrt{H_{o s c} M_{p}}}{T_{d}} \frac{m_{\sigma}^{2} \sigma_{o s c}^{2}}{H_{o s c}^{2} M_{p}^{2}} \geq 5 \times 10^{-5} .
$$

Since the naive bound from nucleosynthesis is $T_{d}>1 \mathrm{MeV}$, one can obtain

$$
\frac{2}{3} \frac{H_{I} \sigma_{o s c} m_{\sigma}^{2}}{2 \pi H_{o s c}^{\frac{3}{2}} M_{p}^{\frac{5}{2}}} \geq 5 \times 10^{-26} .
$$

On the other hand, one can use the lower bound for the curvaton decay rate $\Gamma_{\sigma} \geq \frac{m_{\sigma}^{3}}{M_{p}^{2}}$ to obtain $T_{d} \simeq \sqrt{M_{p} \Gamma} \geq M_{p}\left(m_{\sigma} / M_{p}\right)^{\frac{3}{2}}$, which implies that

$$
\frac{2}{3} \frac{H_{I} \sigma_{o s c} m_{\sigma}^{\frac{1}{2}}}{2 \pi H_{o s c}^{\frac{3}{2}} M_{p}} \geq 5 \times 10^{-5}
$$

The four parameters $\left(H_{I}, \sigma_{o s c}, H_{o s c}, m_{\sigma}\right)$, are the boundary condition that depends on which model one may choose. Here the Hubble parameter during inflation when the observable University leaves the horizon is denoted by $H_{I}$. Considering (2.5) and $r<1$, one finds

$$
\frac{2}{3} \frac{H_{I}}{2 \pi \sigma_{\text {osc }}}>5 \times 10^{-5} .
$$

From eq.(2.9) and (2.7), one finds

$$
H_{I}>10^{-15} \times \frac{H_{o s c}^{\frac{3}{4}} M_{p}^{\frac{5}{4}}}{m_{\sigma}} .
$$


On the other hand, from eq.(2.9) and (2.8) one obtains the following constraint;

$$
H_{I}>10^{-4} \times \frac{H_{o s c}^{\frac{3}{4}} M_{p}^{\frac{1}{2}}}{m_{\sigma}^{\frac{1}{4}}} .
$$

First let us consider a simple example of the boundary condition. We will assume that the mass of the curvaton $m_{\sigma}$ is a constant during the period we are interested in. Then the oscillation of the curvaton field starts when the Hubble parameter falls below the curvaton mass, $H<m_{\sigma}$, which means $H_{o s c} \simeq m_{\sigma}$. As the inequality $H_{I}>H_{\text {osc }}$ always holds, we set a new parameter $\epsilon_{H}$, which is defined as $H_{o s c} \equiv \epsilon_{H}^{2} H_{I}$. Now one can replace $H_{o s c}$ and $m_{\sigma}$ in eq.(2.10) by $H_{I}$ to find

$$
H_{I}>M_{p} \times 10^{-12} \times \epsilon_{H}^{-\frac{2}{5}} \simeq 10^{6} \epsilon_{H}^{-\frac{2}{5}} \mathrm{GeV} .
$$

Following the same arguments, one can find from (2.11),

$$
H_{I}>10^{-8} M_{p} \epsilon_{H}^{2} \simeq 10^{10} \epsilon_{H}^{2} G e V
$$

As is discussed in ref.[10, the bound from eq.(2.13) becomes more strict than (2.12) when $\epsilon_{H}>10^{-2}$. Note that a tiny $\epsilon_{H}$ does not relax the bound. The obtained bound $H_{I}>10^{7} \mathrm{GeV}$ corresponds to the first constraint that was obtained in ref. [10].

In the above arguments we showed that the bound obtained in ref.[10] is derived from an initial condition that fixes the four parameters $\left(H_{I}, \sigma_{o s c}, H_{o s c}, m_{\sigma}\right)$. Because some part of the initial condition is still model dependent, it is interesting to find models that evade the above constraint without introducing fine-tunings or large hierarchy. A way to relax the bound is already suggested in [10]. We think it is instructive to reproduce the argument within our setups and show explicitly how the initial condition is modified in the model. In the "heavy curvaton" scenario, two differences appear in the initial condition. Assuming that there was a phase transition just after inflation, which makes the curvaton mass much larger than the one during inflation, the conditions are modified as follows;

$$
\begin{aligned}
& H_{o s c} \simeq m_{\sigma} \quad \rightarrow \quad H_{o s c} \ll m_{\sigma} \\
& H_{o s c}<H_{I} \quad \rightarrow \quad H_{o s c} \simeq H_{I} .
\end{aligned}
$$

Using the modified initial conditions and (2.10), one can easily find

$$
H_{I}>10^{-56} \frac{M_{p}^{5}}{m_{\sigma}^{4}} .
$$


One can also find another bound from (2.11),

$$
H_{I}>10^{-14} \frac{M_{p}^{2}}{m_{\sigma}}
$$

As is suggested in [10, it might be possible to obtain a preferable bound $H_{I}>O(\mathrm{TeV})$ if the curvaton mass becomes as large as the Planck mass. A huge curvaton mass may be allowed in a conventional supergravity, but is not preferable in the models of large or intermediate extra dimensions. Thus it is still an interesting problem to find models in which the bound is more relaxed.

\section{Multiple inflation}

In the followings we try to find another model whose initial condition significantly lowers the above bound for $H_{I}$. The most promising example will be to assume that the phase transition that induces the curvaton oscillation starts independently of the inflation that produces the spectrum of the perturbation $\delta \sigma$. The easiest way to realize the model is to introduce secondary weak inflation that triggers the required phase transition. ${ }^{3}$

Here we introduce the parameter $H_{e}$ that denotes the Hubble parameter during the last inflation. The spectrum of the perturbation $\delta \sigma$ is produced during the first inflation, the Hubble parameter during which is denoted by $H_{I}$. More explicitly, the parameters follow the conditions, $H_{I} \gg H_{e}, m_{\sigma} \gg H_{o s c}$ and $H_{o s c} \simeq H_{e}$. The bound (2.10) now becomes

$$
H_{I}>10^{-15} \frac{H_{e}^{\frac{3}{4}} M_{p}^{\frac{5}{4}}}{m_{\sigma}} .
$$

Of course, setting $H_{I}=H_{e}=H_{o s c}$, one obtains (2.15) again. The bound (2.11) becomes

$$
H_{I}>10^{-4} \frac{H_{e}^{\frac{3}{4}} M_{p}^{\frac{1}{2}}}{m_{\sigma}^{\frac{1}{4}}} .
$$

Now let us consider a case when $H_{e}=H_{I} \times 10^{-4}$. In this case, (3.2) becomes

$$
H_{I}>10^{-26} \frac{M_{p}^{2}}{m_{\sigma}}
$$

\footnotetext{
${ }^{3}$ In this paper we do not mention how to realize the multiple inflation. Some arguments are given in [5. 11].
} 
which is about $10^{-12}$ times smaller than (2.16). To see exactly what happened in the bound, here we consider a more generic situation. Introducing mass scales $M_{I} \equiv V_{I}^{\frac{1}{4}}=$ $H_{I}^{2} M_{p}^{2}$ and $M_{e} \equiv V_{e}^{\frac{1}{4}}=H_{e}^{2} M_{p}^{2}$, and denoting the ratio by $\epsilon \equiv \frac{M_{e}}{M_{I}}$, one finds from (3.1),

$$
H_{I}>10^{-56} \epsilon^{6} \frac{M_{p}^{5}}{m_{\sigma}^{4}} .
$$

One can also find from (3.2),

$$
H_{I}>10^{-14} \epsilon^{6} \frac{M_{p}^{2}}{m_{\sigma}} .
$$

From eq.(3.4) and eq.(3.5), it is easy to understand why the bound is so sensitive to the ratio $\epsilon$. If the scale of the vacuum energy during the first inflation is only $10^{2}$ times larger than the one during the second inflation, the bound is significantly reduced by the factor of $\left(10^{-2}\right)^{6}$.

\section{Conclusions and Discussions}

In this paper we have examined the constraint on the Hubble parameter during inflation when the curvaton hypothesis is used to explain the observed density perturbation of the Universe. The constraint (2.6) that we have started from produces bounds for the Hubble parameter once the initial conditions are determined by the models. We have found an example of the model that significantly modifies the bound. At least two stages of inflation are required in our scenario. The first produces the spectrum of the perturbation $\delta \sigma$ while the other triggers the phase transition that produces the curvaton mass. Then the ratio of the vacuum energy $\epsilon$ reduces the constraint by the factor of $\epsilon^{6}$, which evades the bound that was discussed in [10].

\section{Acknowledgment}

We wish to thank K.Shima for encouragement, and our colleagues in Tokyo University for their kind hospitality. 


\section{References}

[1] S. Mollerach, Phys.Rev.D42(1990)313; A. D. Linde, V. Mukhanov, Phys.Rev.D56(1997)535; K. Enqvist, M. S. Sloth, Nucl.Phys.B626(2002)395.

[2] T. Moroi, T. Takahashi, Phys.Lett.B522(2001)215; D. H. Lyth, D. Wands, Phys.Lett.B524(2002)5.

[3] "Models of inflation liberated by the curvaton hypothesis", K. Dimopoulos, D. H. Lyth, hep-ph/0209180 M. Giovannini, Phys.Rev.D67(2003)123512, “Tracking curvaton(s)?", hep-ph/0310024

[4] I.Antoniadis, N.A-Hamed, S.Dimopoulos, and G.R.Dvali, Phys.Lett.B436(1998)257; I.Antoniadis, Phys.Lett.B246(1990)377; N.A-Hamed, S.Dimopoulos and G.R.Dvali, Phys.Lett.B429(1998)263.

[5] N. Arkani-Hamed, S. Dimopoulos, N. Kaloper, and J. March-Russell, Nucl.Phys.B567(2000)189; R. N. Mohapatra, A. Perez-Lorenzana, and C. A. de S. Pires, Phys.Rev.D62(2000)105030; A. M. Green and A. Mazumdar, Phys.Rev.D65(2002)105022; D. H. Lyth, Phys.Lett.B448(1999)191; Phys.Lett.B466(1999)85; T. Matsuda, Thys.Rev.D67(2003)083519; JCAP0306(2003)007; Phys.Rev.D68:047702,2003; “ F-term, D-term and hybrid brane inflation", hep-ph/0302078, "Q ball inflation", hep-ph/0309339 M. Axenides, K. Dimopoulos, "Inflation without flat directions", hep-ph/0310194

[6] T.Matsuda, Phys.Lett.B423(1998)35.

[7] G.R. Dvali, G. Gabadadze, Phys.Lett.B460(1999)47; T. Matsuda, Phys.Rev.D66(2002)107301; Phys.Rev.D66(2002)023508; Phys.Rev.D65(2002)107302; Phys.Rev.D64(2001)083512; J.Phys.G27(2001)L103; A. Masiero, M. Peloso, L. Sorbo, and R. Tabbash, Phys.Rev.D62(2000)063515; A.Pilaftsis, Phys.Rev.D60(1999)105023; R.Allahverdi, K.Enqvist, A.Mazumdar and A.P-Lorenzana, Nucl.Phys. B618(2001)377; S. Davidson, M. Losada, and A. Riotto, Phys.Rev.Lett.84(2000)4284.

[8] A.Mazumdar, Nucl.Phys.B597(2001)561, Phys.Rev.D64(2001)027304; A. Mazumdar and A. Perez-Lorenzana, Phys.Rev.D65(2002)107301; R. Allahverdi, K. En- 
qvist, A. Mazumdar, and A. Perez-Lorenzana, Nucl.Phys.B618(2001)277; T.Matsuda, Phys.Rev.D65(2002)103502.

[9] T.Matsuda, Phys.Rev.D65(2002)103501; Phys.Rev.D67(2003)127302.

[10] "Can the curvaton paradigm accommodate a low inflation scale?" D. H. Lyth hep-th/0308110

[11] D.H. Lyth and E. D. Stewart, Phys.Rev.D53(1996)1784; G.R. Dvali, Phys.Lett.B459(1999)489. 